\title{
Global Polarization Theory Overview
}

\author{
Jian-Hua Gao ${ }^{1, *}$ \\ ${ }^{1}$ Shandong Provincial Key Laboratory of Optical Astronomy and Solar-Terrestrial Environment, \\ Institute of Space Sciences, Shandong University, Weihai, Shandong 264209, China
}

\begin{abstract}
We give a brief overview about theory development of spin polarization in relativistic heavy ion collisions, which includes how the polarization could be generated by single scattering, what the polarization could be in equilibrium, how to address some recent puzzles in spin polarization in heavy ion collisions and how much progress we have made in spin hydrodynamics and spin kinetic theory. We will also discuss the possible helicity polarization in relativistic heavy ion collisions.
\end{abstract}

\section{Introduction}

Spin freedom plays an important role to understand the nuclei's structure in low energy nuclear physics. For example, nuclei exhibit extra stability when either the number of proton or neutron is equal to the magic number $2,8,20,28,50,82$ or 126 . The magic numbers can only be explained by adding a spin-orbital coupling term in nuclear shell model. Another example is high spin states in heavy ion fusion evaporation reaction. When two nuclei collide at low energy, they can combine together, exhibit a collective rotation and becomes a high spin state. Such high spin state can be regarded as a rigid rotating body and collective rotation can align the nucleon's angular momentum along the direction of the angular velocity due to Coriolis force, termed as angular momentum alignment. At high-energy heavy ion collisions, the colliding nuclei can not fusion into high spin states like a rigid rotating body any more. Instead, Liang and Wang in [1] first pointed out that the initial orbital angular momentum in non-central heavy ion collisions could be transferred into the momentum or rapidity gradient along the transverse direction. In local comoving frame of the fluid, the colliding particles will carry a local orbital angular momentum along the initial global orbital angular momentum. A natural question is: could this local orbital angular momentum be transformed into the spin angular momentum - spin polarization? As such polarization is always along the initial global orbital angular momentum, it has been given a specific name as global polarization.

\section{Global spin polarization}

Global polarization was first estimated by single scattering in static potential model [1] and then was generalized to realistic two particle scattering with hard thermal loop propagator [2]. The scattering with initial local angular momentum of two particles can be described

\footnotetext{
*e-mail: gaojh@sdu.edu.cn
} 
by the cross section in impact parameter space. In general,the cross section for two particles with impact parameter $\mathbf{x}_{T}$ can be decomposed into two parts

$$
\frac{d \sigma_{s}}{d^{2} \mathbf{x}_{T}}=\frac{d \sigma}{d^{2} \mathbf{x}_{T}}+s \frac{d \Delta \sigma}{d^{2} \mathbf{x}_{T}}, \quad \frac{d \Delta \sigma}{d^{2} \mathbf{x}_{T}} \propto \mathbf{n} \cdot\left(\mathbf{x}_{T} \times \mathbf{p}\right)
$$

where $s= \pm 1$ denotes the particle's spin index. It is obvious that when the polarization direction $\mathbf{n}$ is along the orbital angular momentum, the polarization reaches to maximal value:

The estimation for global polarization at RHIC energy $200 \mathrm{GeV}$ from static potential model is about $-30 \%$ while the observed global polarization from STAR at this energy is consistent with zero within the precision of the measurements [3]. When we generalize the static potential model to hard thermal loop propagator and go beyond the small angle approximation, the estimation can be consistent with the data in the Bjorken scenario at least qualitatively [2]. The most important thing about single scattering model is that it provided us a very intuitive way to understand how the orbital angular momentum can be transformed into spin angular momentum.

Single scattering is only a naive limit, we need consider the multiple scattering process for the realistic heavy ion collisions at high energy. For simplicity, we can consider another naive limit - what the polarization would be if the particles experience multiple scattering so that the system reaches global equilibrium with angular momentum. The spin polarization vector $S^{\mu}(x, p)$ in phase space for spin $1 / 2$ particles in such case can be directly calculated from the density operator at global equilibrium with given angular momentum and the result is given by [4]

$$
S^{\mu}(x, p)=-\frac{1}{8 m}\left(1-n_{F}\right) \epsilon^{\mu \nu \rho \sigma} p_{\nu} \varpi_{\rho \sigma}, \quad \varpi_{\rho \sigma}=-\frac{1}{2}\left[\partial_{\rho}\left(\beta u_{\sigma}\right)-\partial_{\sigma}\left(\beta u_{\rho}\right)\right], \quad \beta=\frac{1}{T}
$$

where $n_{F}$ is Fermi-Dirac distribution, $m$ is the particle's mass and $\varpi^{\rho \sigma}$ is thermal vorticity tensor. We note that the polarization is determined by the thermal vorticity

Although this result is derived from global equilibrium, we expect it should give dominant contribution at local equilibrium. With such assumption, the spin polarization vector at freeze-out hyper-surface in heavy ion collisions can be calculated from

$$
S^{\mu}(p)=-\frac{1}{8 m} \epsilon^{\mu \nu \rho \sigma} p_{v} \frac{\int d \Sigma_{\lambda} p^{\lambda} \varpi_{\rho \sigma} n_{F}\left(1-n_{F}\right)}{\int d \Sigma_{\lambda} p^{\lambda} n_{F}}
$$

In non-central heavy ion collisions, the initial orbital angular momentum could be transferred into global vorticity along this direction which further induce the global polarization. This conclusion is consistent with the result from single scattering model. The calculations for the global polarization based on this expression within different models are all quantitatively consistent with the recent experimental data. The averaged polarization indicate a vorticity of $\omega \approx(9+1) \times 10^{21}$ [5], which is much greater than the vorticity of all other known fluids.

\section{Local spin polarization}

In relativistic heavy ion collisions, the hot system produced after collisions display more complicated vorticity structure than the global vorticity along the direction of initial orbital angular momentum. The simulation indicates that some very interesting local vorticity structure could exist in heavy ion collisions, for example the transverse vorticity exhibits a smoke-ring distribution along the bean momentum axis and the longitudinal vorticity exhibits a quadruple structure in the transverse plane $[6,7]$. These local vorticity structure will lead to the 
local spin polarizaiton, which can be detected by the azimuthal angle dependence of the polarization. However the experimental data shows that both transverse and longitudinal polarization are opposite to the hydrodynamic prediction based on the expression (4). This is so-called local spin puzzle in relativistic heavy ion collisions. This inconsistency can not be solved by the feed-down effect from more heavy hyperons [8, 9]. Recently, some collaborations has proposed that the shear tensor could possibly contribute to the spin polarization. In Refs. $[10,11]$, the authors derive the shear-induced spin polarization (SIP) from chiral kinetic theory or linear response theory

$$
A_{\mathrm{SIP}}^{\mu}=-\beta n_{F}\left(1-n_{F}\right) \frac{p_{\perp}^{2}}{u \cdot p} \epsilon^{\mu v \alpha \rho} u_{\nu} Q_{\alpha}{ }^{\lambda} \sigma_{\rho \lambda}, \quad \sigma_{\rho \lambda}=\frac{1}{2}\left(\partial_{\rho}^{\perp} u_{\lambda}+\partial_{\lambda}^{\perp} u_{\rho}+\frac{2}{3} \partial \cdot u \Delta_{\rho \lambda}\right)
$$

where $p_{\perp}^{\mu}=\Delta^{\mu v} p_{v}, \partial_{\perp}^{\mu}=\Delta^{\mu v} \partial_{v}$, and $Q^{\mu v}=-p_{\perp}^{\mu} p_{\perp}^{v} / p_{\perp}^{2}+\Delta^{\mu v} / 3$. Similarly, the authors in Refs $[12,13]$. also derive the spin polarization from spin-thermal shear coupling by stationary non-equilibrium density operator. With the assumption of isothermal local equilibrium (ILE), they obtain

$$
S_{\xi}^{\mu}=-\frac{1}{4 m} \epsilon^{\mu \nu \sigma \tau} \frac{p_{\tau} p^{\rho}}{p \cdot \hat{t}} \frac{\int_{\Sigma} d \Sigma \cdot p n_{F}\left(1-n_{F}\right) \hat{t}_{\nu} \xi_{\sigma \rho}}{\int_{\Sigma} d \Sigma \cdot p n_{F}}, \quad \xi_{\sigma \rho}=\frac{1}{2}\left[\partial_{\sigma}\left(\beta u_{\rho}\right)+\partial_{\rho}\left(\beta u_{\sigma}\right)\right]
$$

where $\hat{t}$ is the unit vector normal to the hypersurface $\Sigma_{B}$ which corresponds to the time direction in the QGP frame. Although these two methods are slightly different but both shear tensor contribution always shows the same azimuthal angle dependence as experimental result. In the "strange memory" scenario of the first method and with the assumption of isothermal local equilibrium of the second method, the total results from shear tensor and vorticity tensor give the right trend with the experimental tensor.

Another spin puzzle in heavy ion collisions is relevant to spin alignment of vector meson. In the recombination scenario, naive estimation gives the spin alignment should be squared order of hyperon's global polarization $P_{H}^{2} \sim 10^{-4}$ while the experiment result is the order $10^{-1}$. Besides, the $\phi$ meson can take a significant positive deviation from $1 / 3$ which is very different from $K^{* 0}$ meson. It has been proposed that the existence of a mean field of the $\phi$ meson generated in heavy-ion collisions can give a possible mechanism to result in positive deviation from $1 / 3$ [14]. It has been also found that the spin alignment does not necessary signal the global polarization but may also originate from local spin polarization [15]. The strong alignment measured by STAR and ALICE which is not consistent with hyperon polarization might be due to the fact that the global polarization is very small while local polarization fluctuation is very large.

\section{Spin hydrodynamics and spin kinetic theory}

Single scattering and global equilibrium are two opposite limits, spin hydrodynamics or the spin kinetic theory can connect these two limits and gives more realistic description for the spin polarization in heavy ion collisions. In recent years, a lot of important progresses have been made in this direction.

In the chiral system, the particle's spin always coincides with the momentum and the spin is not a dynamical variable, spin hydrodynamics can be reduced into anomalous hydrodynamics $[16,17]$, which had been established very well,

$$
\partial_{\mu} T^{\mu v}=F^{v \lambda} j_{\lambda}, \quad \partial_{\mu} j^{\mu}=0, \quad \partial_{\mu} j_{5}^{\mu}=C E \cdot B
$$


where the information for spin has been encoded in the axial currrent $j_{5}^{\mu}$. Similarly, the spin kinetic theory for chiral particles can be reduced into chiral kinetic theory in which only right-handed or left-handed particle distribution function $f$ is independent $[18,19]$,

$$
p \cdot \partial f=\int_{B C D} C_{A B C D}[f]\left(1-\int_{B^{\prime} C^{\prime} D^{\prime}} \frac{\partial}{\partial f} C_{A B^{\prime} C^{\prime} D^{\prime}} \frac{\bar{\Delta} \cdot \bar{n}^{\prime}}{p \cdot \bar{n}^{\prime}}\right)
$$

where the collision kernel $C_{A B C D}$ is defined by the difference between the rate of collisions $C_{A B C D} \equiv W_{C D \rightarrow A B}-W_{A B \rightarrow C D}, \bar{n}^{\prime}$ is the no-jump frame in the collison $A B^{\prime} \leftrightarrow C^{\prime} D^{\prime}$ and $\bar{\Delta}$ denotes tunnelinglike motion in nonlocal collisions. The recent simulation [20] from chiral kinetic equation has shown that the non-local collision from angular momentum conservation plays a very important role to reproduce the consistent result of local polarization.

When the particle has a finite mass, the spin will become a dynamical variable and we need extra variables describe the spin evolution. In spin hydrodynamics, spin chemical potential $\omega^{\mu v}$ has been introduced into relativistic hydrodynamics to describe the spin evolution. In addition to the conservations of energy-momentum and charge, spin dynamics is determined by the conservation of angular momentum [21],

$$
\partial_{\mu} T^{\mu v}=0, \quad \partial_{\mu}\left(n u^{\mu}\right)=0, \quad \partial_{\lambda} S^{\lambda, \mu v}=0 .
$$

In the ideal spin hydrodynamics, the spin tensor $S^{\lambda, \mu v}$ is proportional to spin chemical potential $S^{\lambda, \mu \nu} \propto u^{\lambda} \omega^{\mu \nu}$. However, from effective field theory, even ideal spin hydrodynamics requires non-dissipative dynamics at higher order than second order, which indicates very non-triviality in spin hydrodynamics. In the regime of ideal spin hydrodynamics, we cannot deal with the spin generation and dissipation. In order to address this problem, we must go beyond ideal spin hydrodynamics and use viscous spin hydrodynamics. Viscous spin hydrodynamics can be formulated from entropy-current analysis and constrain possible constitutive equations of a spin current and the antisymmetric part of canonical energy-momentum tensor [22].

$$
\partial_{\lambda} S^{\lambda, \alpha \beta}=T^{\beta \alpha}-T^{\alpha \beta} \equiv-2 T_{A}^{\alpha \beta}
$$

where antisymmetric part of energy momentum tensor $T_{A}^{\alpha \beta}$ is given by

$$
T_{A}^{\alpha \beta}=-2 \lambda\left(-u \cdot \partial u^{[\mu}+\beta \partial_{\perp}^{[\mu} \beta^{-1}+4 u_{\rho} \omega^{\rho[\mu}\right) u^{\nu]}-2 \gamma\left(\partial_{\perp}^{[\mu} u^{\nu]}-2 \Delta_{\rho}^{\mu} \Delta_{\sigma}^{v} \omega^{\rho \sigma}\right)
$$

where $\lambda$ and $\gamma$ are new transport coefficients associated with spin transport. The viscous spin hydrodynamics can be also derived from classical kinetic equation at relaxation time approximation and the spin chemical potential satisfies

$$
\dot{\omega}^{\mu v}=D_{\Pi}^{\mu v}(\partial \cdot u)+\left(\nabla^{\alpha} \frac{\mu}{T}\right) D_{n}^{[\mu v]}{ }_{\alpha}+D_{\pi \lambda}^{[v} \sigma^{\lambda \mu]}+D_{\Sigma 1}^{\alpha} \nabla^{[\mu} \omega_{\alpha}^{v]}+D_{\Sigma 2}^{[\mu v] \alpha} \nabla^{\lambda} \omega_{\alpha \lambda}
$$

where the explicit expressions for $D$ coefficient are given by [23]. The spin hydrodynamics has also been derived from chiral kinetic theory with realistic collision term [24] and the results is more complicated than these simplified version.

The spin kinetic equation for massive fermions in background field approximation without collision term can be directly derived from Wigner function approach. However the kinetic equation can take different forms which depends on the different independent distribution function. Here we will only present one form given in [25] in which scalar distribution $\mathcal{F}$ and spin vector distribution $\mathcal{A}$ have been chosen as independent variables.

$$
\begin{aligned}
\left(\nabla_{t}+\mathbf{v} \cdot \boldsymbol{\nabla}\right) \mathcal{F} & =-\frac{\hbar}{2 m E_{p}}\left[(\mathbf{B}+\mathbf{E} \times \mathbf{v}) \nabla_{\mathbf{v}}-(\mathbf{B} \cdot \mathbf{v}) \nabla_{\mathbf{v}} \mathbf{v}\right] \cdot \mathcal{A}, \\
\left(\nabla_{t}+\mathbf{v} \cdot \boldsymbol{\nabla}\right) \mathcal{A} & =\mathbf{B} \times \mathcal{A}-\mathbf{E}(\mathbf{v} \cdot \mathcal{A})-\frac{\hbar}{2 m E_{p}}(\mathbf{B}+\mathbf{E} \times \mathbf{v}) \nabla_{\mathbf{v}} \mathcal{F},
\end{aligned}
$$


where $\mathbf{v}=\mathbf{p} / E_{p}, \nabla_{t}=\partial_{t}+\mathbf{E} \cdot \boldsymbol{\nabla}_{p}, \boldsymbol{\nabla}=\boldsymbol{\nabla}_{x}+\mathbf{B} \times \boldsymbol{\nabla}_{p}$ and $\nabla_{\mathbf{v}}=\mathbf{v} \cdot \boldsymbol{\nabla}+E_{p} \overleftarrow{\boldsymbol{\nabla}}_{x} \cdot \boldsymbol{\nabla}_{p}$. For spin kinetic theory with collision term derived from quantum field theory, some attempt has been made and the final result is much more complicated than the spin kinetic equation without collision term. Further work is needed for numerical simulation. However the non-local collision term can be derived explicitly [26]

$$
\begin{aligned}
& p \cdot \partial \tilde{f}(x, p, s)=\int d \Gamma_{1} d \Gamma_{2} d \Gamma^{\prime} \tilde{W}\left[\tilde{f}\left(x+\Delta_{1}, p_{1}, s_{1}\right) \tilde{f}\left(x+\Delta_{2}, p_{2}, s_{2}\right)\right. \\
& \left.-\tilde{f}(x+\Delta, p, s) \tilde{f}\left(x+\Delta^{\prime}, p^{\prime}, s^{\prime}\right)\right]+\int d \Gamma_{2} d S_{1}(p) M \tilde{f}\left(x+\Delta_{1}, p_{1}, s_{1}\right) \tilde{f}\left(x+\Delta_{2}, p_{2}, s_{2}\right)
\end{aligned}
$$

where $\Delta, \Delta^{\prime}, \Delta_{1}$ and $\Delta_{2}$ are position shifts which exhibit non-local collision term explicitly,

\section{Helicity polarization in relativistic heavy ion collisions}

In addition to the global or local spin polarization along the global orbital angular momentum or beam momentum, another possible spin polarization along the momentum of final hadrons - helicity polarization is also discussed recently $[27,28]$. The helicity polarization can be induced by both helicity charge and vorticity tensor,

$$
\mathcal{A}^{\mu}(x, p)=\frac{p^{\mu}}{E_{p}} \sum_{\lambda} \lambda f_{\lambda}-\frac{\epsilon^{\mu \nu \rho \sigma} p_{\nu} \varpi_{\rho \sigma}}{4 E_{p}} \sum_{\lambda} f_{\lambda}\left(1-f_{\lambda}\right),
$$

where the first term is pure helicity charge contribution and the second term is usual vorticity contribution. It is easy to show that only the spatial components of vorticity tensor are involved for the helicity polarization. Even with the initial zero helicity charge, the helicity charge can be redistributed due to the chiral separate effect from vorticity for the viscous fluid

$$
\partial_{\mu}\left(n_{5} u^{\mu}\right)=-\frac{T}{6 s} \epsilon_{\mu \lambda \rho \sigma} u^{\lambda} \partial^{\rho} u^{\sigma} \partial_{\nu} \pi^{\mu \nu} .
$$

where $n_{5}$ is helicity charge density, $\pi^{\mu \nu}$ is dissipative tensor and $s$ is entropy density.

In relativistic heavy ion collisions, the initial helicity charge could exist due to classical color longitudinal fields or QCD sphaleron transitions in the quark gluon plasma. Such initial helicity charge indicates the local parity violation in QCD. Recently it has been proposed that this initial helicity charge can be measured by the correlation of the hyperons' helicity polarization $[27,28]$. The helicity alignment for vector meson from helicity charge can contribute to the spin alignment along $x, y$ and $z$ axis,

$$
\bar{\rho}_{00}^{x}=\frac{1}{3}+\frac{1}{4} \Delta+\frac{3}{4} v_{2} \Delta, \quad \bar{\rho}_{00}^{y}=\frac{1}{3}+\frac{1}{4} \Delta-\frac{3}{4} v_{2} \Delta, \quad \bar{\rho}_{00}^{z}=\frac{1}{3}-\frac{1}{2} \Delta
$$

where $\Delta=\rho_{00}-1 / 3$ is deviation from $1 / 3$ for the helicity density matrix element $\rho_{00}$ and $v_{2}$ is elliptic flow of the momentum distribution of vector meson.

\section{Summary and outlook}

Spin plays an important role in nuclear structure at low energy. The observation of global polarization opens a new avenue to understand the quark gluon plasma at high energy. With the upcoming running of NICA, FAIR, J-PARK and HIAF, we can expect that a big picture of spin in nuclear physics would be coming. Local spin polarization for hyperons and spin alighment for vector mesons are both still in debate and quantitative prediction by spin hydrodynamics or spin kinetic theory is needed. Helicity polarization would give us another new probe to detect the spin polarization in heavy ion collisions. 


\section{Acknowledgments}

This work was supported in part by the National Natural Science Foundation of China under Grant Nos. 11890710, 11890713 and 11475104.

\section{References}

[1] Z. T. Liang and X. N. Wang, Phys. Rev. Lett. 94, 102301 (2005) [erratum: Phys. Rev. Lett. 96, 039901 (2006)]

[2] J. H. Gao, S. W. Chen, W. t. Deng, Z. T. Liang, Q. Wang and X. N. Wang, Phys. Rev. C 77, 044902 (2008)

[3] B. I. Abelev et al. [STAR], Phys. Rev. C 76, 024915 (2007) [erratum: Phys. Rev. C 95, no.3, 039906 (2017)]

[4] F. Becattini, V. Chandra, L. Del Zanna and E. Grossi, Annals Phys. 338, 32-49 (2013)

[5] L. Adamczyk et al. [STAR Collaboration], Nature 548, 62 (2017).

[6] F. Becattini and I. Karpenko, Phys. Rev. Lett. 120, no.1, 012302 (2018)

[7] X. L. Xia, H. Li, Z. B. Tang and Q. Wang, Phys. Rev. C 98, 024905 (2018)

[8] X. L. Xia, H. Li, X. G. Huang and H. Z. Huang, Phys. Rev. C 100, no.1, 014913 (2019)

[9] F. Becattini, G. Cao and E. Speranza, Eur. Phys. J. C 79, no.9, 741 (2019)

[10] S. Y. F. Liu and Y. Yin, JHEP 07, 188 (2021)

[11] B. Fu, S. Y. F. Liu, L. Pang, H. Song and Y. Yin, [arXiv:2103.10403 [hep-ph]].

[12] F. Becattini, M. Buzzegoli and A. Palermo, Phys. Lett. B 820, 136519 (2021)

[13] F. Becattini, M. Buzzegoli, A. Palermo, G. Inghirami and I. Karpenko, [arXiv:2103.14621 [nucl-th]].

[14] X. L. Sheng, L. Oliva and Q. Wang, Phys. Rev. D 101, no.9, 096005 (2020)

[15] X. L. Xia, H. Li, X. G. Huang and H. Zhong Huang, Phys. Lett. B 817, 136325 (2021)

[16] D. T. Son and P. Surowka, Phys. Rev. Lett. 103, 191601 (2009)

[17] S. Pu, J. h. Gao and Q. Wang, Phys. Rev. D 83, 094017 (2011)

[18] M. A. Stephanov and Y. Yin, Phys. Rev. Lett. 109, 162001 (2012)

[19] J. Y. Chen, D. T. Son and M. A. Stephanov, Phys. Rev. Lett. 115, no.2, 021601 (2015)

[20] S. Y. F. Liu, Y. Sun and C. M. Ko, Phys. Rev. Lett. 125, no.6, 062301 (2020)

[21] W. Florkowski, B. Friman, A. Jaiswal and E. Speranza, Phys. Rev. C 97, no.4, 041901 (2018)

[22] K. Hattori, M. Hongo, X. G. Huang, M. Matsuo and H. Taya, Phys. Lett. B 795, 100-106 (2019)

[23] S. Bhadury, W. Florkowski, A. Jaiswal, A. Kumar and R. Ryblewski, Phys. Rev. D 103, no.1, 014030 (2021)

[24] S. Shi, C. Gale and S. Jeon, Phys. Rev. C 103, no.4, 044906 (2021)

[25] J. H. Gao and Z. T. Liang, Phys. Rev. D 100, no.5, 056021 (2019)

[26] N. Weickgenannt, E. Speranza, X. 1. Sheng, Q. Wang and D. H. Rischke, Phys. Rev. Lett. 127, no.5, 052301 (2021)

[27] F. Becattini, M. Buzzegoli, A. Palermo and G. Prokhorov, [arXiv:2009.13449 [hep-ph]].

[28] J. H. Gao, [arXiv:2105.08293 [hep-ph]]. 